\title{
CONTEXTO EDUCATIVO Y VULNERABILIDAD
}

\author{
Nadia Semenova Moratto Vásquez $z^{1}$ \\ Daniela Sánchez Acosta ${ }^{2}$
}

\section{Resumen}

Nombrar a la psicología como educativa, demarca diferencias conceptuales y epistemológicas desde diversas disciplinas, tales como la pedagogía, la educación, la psicología, entre otras; así como delimita un campo de acción que entreteje diversos caminos que implican racionalidades individuales, grupales y de comunidad, diferenciales en contenido y metodología. Si bien lo anterior constituye un contexto ineludible, el texto que se presenta propone más que la discusión epistémica desde la noción de campos, una reflexión por aquellos aspectos inherentes a la disciplina de la psicología puesta en acción, en el contexto de la educación. Se abordan, así, tres categorías sustanciales y engranadas: las diferencias individuales, los factores relativos a las situaciones educativas y la conceptualización de riesgo y vulnerabilidad en este contexto. En relación con las diferencias individuales, se piensa en procesos propios, no sólo de la modificación del comportamiento o los mecanismos de aprendizaje, sino también de la evaluación del nivel propio de desarrollo evolutivo, las características aptitudinales, afectivas y rasgos de personalidad de niños y adolescentes. En tanto los factores relativos a las situaciones educativas, se discriminan aspectos que, de forma directa o indirecta, ejercen una influencia en el proceso anteriormente descrito, bien sean las características del profesor, las relaciones interpersonales propias de los grupos, las condiciones materiales y medios de enseñanza en general, así como las intervenciones pedagógicas implementadas. Por último, respecto a riesgo y vulnerabilidad, se opta por comprender conceptual y contextualmente estos términos, particularizando el escenario colombiano y cómo, desde este lugar, es necesario generar

$1 \quad$ Candidata a Doctor en Ciencias Sociales, UPB Medellín. Magíster en Psicología. Grupo de Investigación Psicología, Salud y Sociedad. Universidad CES. Medellín, Colombia.

2 Magíster en Salud Mental de la Niñez y la Adolescencia. Grupo de Investigación Psicología, Salud y Sociedad. Universidad CES. Medellín, Colombia 
nuevas líneas de trabajo y preguntas de investigación que involucren la realidad de la dinámica social propia. Para dar cumplimiento a lo anterior, se realizó una investigación documental abordando los conceptos de psicología educativa, psicología de la educación, diferencias individuales, vulnerabilidad y contexto educativo. Una vez realizado el proceso de búsqueda y sistematización de información, se agruparon los hallazgos en tres categorías a saber: a) Psicología educativa y contexto escolar; b) los sujetos inmersos en el contexto educativo: diferencias individuales; y c) las necesidades del contexto escolar y vulnerabilidad social.

Palabras claves: psicología educativa, diferencias individuales, riesgo psicosocial y vulnerabilidad.

\section{Abstract}

Naming psychology as educational, demarcates conceptual and epistemological differences from various disciplines, such as pedagogy, education, psychology, among others; as well as it delimits a field of action that interweaves diverse paths that imply individual, group and community rationalities, differentials in content and methodology. Although the above constitutes an inescapable context, the text that is presented proposes more than the epistemic discussion from the notion of fields, a reflection of those aspects inherent to the discipline of psychology put into action, in the context of education. Thus, three substantial and interlinked categories are addressed: individual differences, factors related to educational situations and the conceptualization of risk and vulnerability in this context. In relation to individual differences, one thinks about own processes, not only of the modification of the behavior or the learning mechanisms, but also of the evaluation of the own level of evolutionary development, the aptitudes, affective characteristics and personality traits of children and teenagers. As factors related to educational situations, aspects that directly or indirectly exert an influence on the process described above are discriminated, whether the characteristics of the teacher, the interpersonal relationships of the groups, material conditions and means of teaching in general, as well as the pedagogical interventions implemented. Finally, regarding risk and vulnerability, we choose to understand these terms conceptually and contextually, particularizing the Colombian scenario and how, from this place, it is necessary to generate new lines of work and research questions that involve the reality of our own social dynamics. . In order to comply with the above, a documentary research was carried out addressing the concepts of educational psychology, educational psychology, 
individual differences, vulnerability and educational context. Once the information search and systematization process was carried out, the findings were grouped into three categories, namely: a) Educational psychology and school context; b) the subjects immersed in the educational context: individual differences; and c) the needs of the school context and social vulnerability.

Key words: educational psychology, individual differences, psychosocial risk and vulnerability

\section{Introducción}

Durante las últimas décadas, las complejas situaciones que se viven en los contextos educativos han generado un interés por parte de diferentes disciplinas para comprender las variadas y multifacéticas relaciones que se entretejen en su interior. En este sentido, pensar en cómo la psicología educativa, como campo permeado por diversas perspectivas teóricas, ha contextualizado, conceptualizado e intervenido diversos fenómenos del contexto escolar, se constituye en una apuesta por esbozar cómo se piensan asuntos como la vulnerabilidad en el contexto colombiano, específicamente sus repercusiones para el ámbito educativo, propósito particular del capítulo que se presenta.

De manera inicial, se propone un marco conceptual sobre la psicología educativa y el contexto escolar, la cual —a pesar de que su constitución como disciplina se dio desde hace más de un siglo_- aún se debate sobre la relación entre la educación y la psicología. Para ello, se realiza un recorrido sobre dicha área del conocimiento, retomando los que se consideran los principales hitos históricos, definiendo sus principios epistemológicos y sus diseños e intereses investigativos, los cuales guían las apuestas en torno a los conceptos claves, entre ellos el de vulnerabilidad en el contexto escolar.

Posteriormente, en términos de contenidos de la psicología educativa, se abordarán sus dos categorías generales. En primer lugar, aquellos aspectos relativos a las diferencias individuales: nivel de desarrollo evolutivo, características aptitudinales, factores emocionales —como la motivación y las actitudes - y de personalidad — entre las que se podrían nombrar el nivel de ansiedad, procesos de cambio comportamental, autoconcepto, mecanismos de aprendizaje, nivel y estructura de los conocimientos previos, 
sistema de valores, sistema de creencias y pautas de comportamientos interpersonales-, entre otros. En segundo lugar, los factores relativos a las situaciones educativas directa o indirectamente responsables de los anteriores factores. En estos se pueden citar las características del profesor (capacidad intelectual, conocimiento de la materia, capacidad pedagógica, rasgos de personalidad, características afectivas), los factores de grupo y sociales (relaciones interpersonales), las condiciones materiales (materiales didácticos y medios de enseñanza en general) y las intervenciones pedagógicas (métodos de enseñanza). Elementos del contexto escolar susceptibles de análisis frente a lo referido como vulnerabilidad.

\section{Psicología educativa y contexto escolar}

Nombrar a la psicología como educativa, tiene una finalidad concreta que hace referencia a uno de los aspectos claves por los cuales en la actualidad esta cuenta con un papel fundamental en la sociedad, es decir, la psicología tiene como función transmitir conocimiento a la población y encargarse de sensibilizar, afianzar y desarrollar estrategias que permitan el crecimiento humano, a través de la enseñanza de nuevas y mejores formas de relación entre estos.

No obstante, cuando se habla de psicología educativa se hacen necesarias otras aclaraciones que, si bien cumplen implícitamente con el objetivo anteriormente mencionado, las tareas o funciones concretas se complejizan al unir dos disciplinas que, aunque propendan por un propósito similar, sus orígenes, técnicas de acercamiento, intervención y estudio son diferentes y constituyen, en sí mismos, el interés que despierta entender cómo la psicología interviene en el contexto educativo.

Los primeros interrogantes sobre la psicología de la educación se remontan a los inicios de la filosofía griega con Aristóteles y Platón, y continúan a la par con los comienzos de la psicología con autores como Binet, Catell, Hall y W. James. Históricamente, se plantea que ya para el año 1880, Louisa Pearson Hopkins escribía acerca de reconocer la importancia de la psicología para la educación: "Sería tan absurdo tratar de educar a los jóvenes sin tener conocimiento de (...) la psicología; como intentar producir una 
sonata cuando se ignora el fenómeno del sonido" (Hopkins, 1886, p.3, citado por Glover \& Bruning, 1990).

Es así como esta disciplina de acercamiento e intervención al hombre y perspectiva de análisis para comprender como este se desenvuelve en un ambiente determinado, ha estado presente por más de un siglo. En términos de línea de tiempo, podría situarse como período clave el año de 1890, puesto que desde allí aparecen figuras importantes con el propósito de lograr objetivar los procesos de enseñanza y evaluación de alumnos, y concientizar a la comunidad de que solo a través de la investigación cuantitativa podrían obtenerse resultados y progreso (Bueno, 2004).

En esta medida, se encuentran diversos autores que dieron impulso a esta disciplina, como es el caso de Galton (1822-1911) y su invención de los tests para medir la inteligencia; Cattell (1860-1944) quien, si bien no se centró en la psicología educativa como tal, su énfasis en el estudio de las diferencias individuales constituye hoy día un propósito obligado de investigación; Dewey (1859-1952) y su idea de encontrar una ciencia que hiciera puente entre la psicología y la práctica educativa; y los estudios sobre el comportamiento humano de J. Watson (1960), con sus posteriores teorías del aprendizaje, por citar algunos.

Según Bueno (1993), el nacimiento, propiamente dicho, de la psicología de la educación se encuadra entre 1900 y 1908, con la aparición e influencia determinante de dos figuras — Thorndike y Judd-, quienes concentran la problemática educativa alrededor del aprendizaje y la lectura. Por un lado, Thorndike, desde su pretensión de unir ambos mundos disciplinares en una ciencia completa, optó por una interpretación científica reduccionista de la psicología de la educación, situándola en el campo de las ciencias exactas y definiéndola como un cuerpo de principios inducidos de los hallazgos descubiertos por la investigación experimental. Por su parte, Judd destaca sus cuatro líneas de trabajo: el estudio de la lectura, la formalización y discusión de los problemas psicológicos que surgen en la enseñanza, el trabajo experimental sobre el número y la psicología social. También en este período de tiempo cobra especial relevancia Terman, quien publica investigaciones sobre niños superdotados y su obra La medida de la inteligencia. 
Siendo así como, desde los inicios de la psicología educativa, surge como respuesta una psicología de la educación que aspire a descubrir leyes y causas que rigen la conducta humana, para poderla predecir y controlar, pero sin olvidar que la conducta humana, además de su vertiente externa manifiesta sometida a cuantificación, predicción, y medida, encierra otra vertiente interior que alberga significados y propósitos, más que causas, y que exige una explicación o interpretación más que una estrategia de control (Beltrán \& Bueno, 2004).

En este sentido, podría mencionarse que el período de afianzamiento de la psicología educativa, incluye los años desde 1918 a 1941, tiempo en el cual se producen tres sucesos importantes: la aplicación de pruebas psicológicas a los soldados del ejército norteamericano; la creación del Consejo Americano de Educación y la publicación de los primeros materiales de tests. Siendo así como en las primeras décadas del siglo XX las áreas de investigación experimental del aprendizaje, los estudios de medida de las diferencias individuales y la psicología del niño, comienzan a nutrir a la psicología de la educación, constituyéndose, en 1940, con total convencimiento de que esta, basada en estas tres áreas, permitiría que la pedagogía alcance definitivamente un estatuto científico. Alrededor de esta posición, surgen diferentes definiciones propuestas por Ausbel (1969), Secdas (1970), Bigs (1976), Glaser (1973), Clifford (1984), Castanedo (1998), entre otras.

Bajo este panorama, el complejo y diverso estudio de la psicología conlleva a pensar la inclusión de distintos puntos de referencia epistemológica, asunto que, desde la década del 50, ha llevado a la psicología de la educación a estar en un lugar privilegiado en el marco de las ciencias sociales, al menos en lo que respecta al campo de la investigación: La orientación cognitiva, con producciones como las de Gagné (1967) y el aprendizaje y las diferencias individuales; Resnick (1976) y la inteligencia; Lahr (1976) y el conocimiento e la interacción; Lesgold (1978) y la psicología cognitiva, el diseño y control de la instrucción; Snow (1980) y la actitud, el aprendizaje yla instrucción. Y, por otro lado, la perspectiva conductista y el enfoque psicosocial, que resaltan el funcionamiento social de los individuos y los grupos en el ambiente escolar, intentando resolver sus problemas, al aplicar los principios psicosociales a los problemas educativos. Finalmente, 
el punto de vista ecológico, con postulados realizados por Barker (1968), Benner (1976) y Moos $(1974,1979)$.

Hasta este punto, se hace necesario aclarar que, si bien se han nombrado eventos importantes que han dado inicio a una prometedora disciplina, se encuentran a su vez grandes detractores de la misma, puesto que, se centran en debatir la relación entre la educación y la psicología y, por ende, sobre cuál debe ser la definición concreta y correcta de ésta.

Referente a ello, Ausbell (1968), citado por Coll, Palacio y Marchesi (1990), menciona que la psicología de la educación ha dejado mucho que desear por la falta de respuesta a las importantes expectativas que se habían versado sobre ella. No es, pues, de extrañarse que esta falta de imagen como disciplina haga que algunos autores se planteen su existencia como tal. A su vez, Beltrán (1996) menciona que la causa real de las adversidades a las que se ha visto enfrentada la psicología educativa, estriba principalmente en el hecho de que desde sus inicios se optó por una interpretación científica reduccionista que pretendía ubicarse en el campo de las ciencias exactas, que no tuvo en cuenta el contexto natural del hombre y lo que a este le influye.

Los comentarios anteriores se vieron claramente reflejados en la década de los cuarenta, donde la psicología educativa comienza a experimentar un importante retroceso e incluso se pensó en su total declive, motivado por diversos factores: falta de un campo propio, invasión al poco espacio que tenía de diversas disciplinas y escasa investigación científica, por mencionar algunas.

Ahora bien, con el fin de aproximarse a un acercamiento conceptual de la psicología educativa, en este punto se hace necesario realizar una definición de la misma. No obstante, se encuentra una dificultad para definir su contenido, dado que se trata de una disciplina resultado de la confluencia de dos campos de estudio diferentes. Además, desde su aparición, se han añadido a su estudio y ámbito de intervención diversos elementos de otras ciencias.

Apostándole a una definición, se encuentra a Ausbel, quien, en el año 1969, define a la psicología educativa de la siguiente forma:

La psicología de la educación es una disciplina aplicada, pero no es la psicología general aplicada a los problemas educativos, 
de igual modo que la ingeniería mecánica no es la física general aplicada a los problemas del diseño de máquinas. O la medicina no es la biología general aplicada a los problemas de diagnóstico, de curación y de prevención de las enfermedades humanas. En estas últimas disciplinas, las leyes generales que tienen su origen en las disciplinas básicas no se aplican al dominio de los problemas prácticos; más bien existe una teoría separada de carácter aplicado que es exactamente tan básica como la teoría de las disciplinas básicas, pero que está enunciada en un nivel inferior de generalidad y posee una relevancia más directa para los problemas aplicados en sus respectivos campos (citado por Coll, Palacio \& Marchesi, 1990).

Un año más tarde, Secadas (1970, citado por Coll et al., 1990) plantea que la psicología de la educación es una rama de la psicología que posee como objeto de estudio la conducta, y, como ciencia aplicada, el estudio de la conducta en situaciones educativas, es decir, la conducta que cambia como resultado del proceso de enseñanza-aprendizaje. Ante esto, surgen algunas propuestas epistemológicas a la psicología de la educación, entre estas la de Biggs (1976) quien crea un neologismo "educología" como ciencia que estudia el logos de la educación (educ= de educational y ology = de psychology), y que integraría también las aportaciones de otras áreas del conocimiento como la filosofía, sociología, historia, etc. Este autor elabora, así, una ciencia integrada de la educación en lugar de considerar diversas ciencias de la educación, entre las cuales estaría la psicología de la educación.

En esta misma década, Glaser (1973-1976) la propone como una disciplina puente entre la psicología y la educación, con un objeto de estudio, unos métodos y unos marcos teóricos y conceptuales propios. Disciplina tecnológica que no puede entenderse al margen de las ciencias básicas correspondientes. Por otra parte, Clifford (1984) define a la psicología educativa como el uso de los métodos de la psicología para estudiar el proceso educativo y factores que en él acontecen. En este sentido, la psicología educativa no configuraría un ámbito propio de conocimiento, sino consecuencia de una variedad claramente seleccionada de los principios y explicaciones que proporcionan otras áreas de la psicología, tales como la 
psicología del aprendizaje, psicología del desarrollo, psicología diferencial, psicología motivacional, psicología social y psicología general.

En contraposición con lo anterior, puede observarse la propuesta de Coll et al. (1990), quienes entienden la psicología de la educación como una ciencia aplicada, ya que no solo busca conocimientos sino también fines prácticos, no se limita a ser un mera y simple psicología general aplicada a los problemas educativos, ni una tecnología que trata de traducir en términos operativos los principios generales de la ciencia de la conducta, porque si fuera así, carecería de un lugar y espacio específico en el mapa de las ciencias. La psicología educativa, entonces, debe investigar problemas educativos en el nivel de complejidad en el que se plantean, es decir, ser una ciencia que estudia la conducta que se produce en situaciones educativas.

Apoyando esta posición, Bueno y Castanedo (1998), manifiestan en los años 90 que la psicología de la educación no debe limitarse a trasponer al ámbito educativo el conocimiento ya elaborado por la investigación psicológica, sino que, por el contrario, debe realizar contribuciones originales teniendo en cuenta, al mismo tiempo, los principios psicológicos y las características de los procesos educativos.

En síntesis, se rechaza la consideración de la psicología de la educación como un simple campo de aplicación de la psicología, y se insiste en la necesidad de atender simultáneamente a los procesos psicológicos y a las características de las situaciones educativas, así como en la necesidad de subrayar el carácter aplicado de la disciplina. La psicología de la educación partiría, entonces, de los mismos paradigmas explicativos que el resto de las disciplinas psicológicas en una relación de interdependencia e interacción. En tanto disciplina educativa, la psicología de la educación debe encargarse de estudiar los procesos educativos en una triple dimensión: Térica o explicativa (proporciona modelos interpretativos de los procesos de cambio comportamental provocados por las situaciones educativas), tecnológicoproyectiva (contribuye al diseño de situaciones educativas que aspiran a promover unos determinados procesos de cambio comportamental), y Técnico-práctica (integra los elementos anteriores con el fin de aportar soluciones a los problemas planteados por la puesta a punto y la realización de actividades educativas). Además, debe contribuir con sus aportaciones a una mejor comprensión, planificación y mejora de los procesos educativos, en una perspectiva multidisciplinar. 
Sin embargo, se debe reconocer que, independiente de las definiciones que durante las diferentes épocas o niveles de estudio se han dado, se hace necesario conceptualizar la psicología educativa tomando como punto de partida desde dónde se abordan las situaciones vividas por los seres humanos en el contexto educativo, es decir, a partir de prestar atención al contexto histórico y natural del hombre. Una de estas miradas es realizada por el campo cognitivo, desde donde se pretende predecir y controlar la conducta, pero, también, y sobre todo, explicarla a través de diferentes mecanismos como lo es el modelo del procesamiento de la información, donde los cambios percibidos en las aulas no se atribuyen específicamente a factores o sucesos externos, sino a cómo las estructuras mentales internas operan y dan significado a las vivencias.

Por otro lado, desde un enfoque psicosocial, se resalta el funcionamiento social de los individuos y los grupos en el ambiente escolar, intentando resolver sus problemas, aplicando los principios psicosociales a los problemas educativos. Por tanto, no solo se consideran las características sociales del estudiante como las actitudes, valores, expectativas y relaciones interpersonales, sino que también se intenta comprobar el modo en que afectan dichas relaciones y particularidades, la conducta de los estudiantes.

Por último, desde un punto de vista ecológico, Bronfenbrenner (1979), considera que se hace esencial considerar la especificidad del ambiente o escenario de la conducta, pero, sobre todo, la percepción e interpretación que de la misma hace cada sujeto.

Reflexionando en torno a las anteriores posturas, podría citarse a Beltrán (1996) quien, en el proceso de definir la psicología educativa, concluye que, definitivamente su tema central de estudio es el aprendizaje y sus procesos, pero que no se desconoce la incursión de esta disciplina en temas como las aptitudes, el desarrollo y, sobre todo, las diferencias individuales.

A partir de esto, se logra vislumbrar un quehacer de la psicología educativa que, si bien hasta ahora se ha centrado en el proceso de enseñanza-aprendizaje, logra adentrarse en cuatro puntos esenciales (Beltrán, 1987, citado por Bueno \& Castanedo, 1998): El que aprende, es decir, de sus procesos de crecimiento, de aprendizaje y de las diferencias individuales de este, tales como su inteligencia, creatividad, personalidad, 
autoesquemas y todos aquellos elementos intrínsecos y extrínsecos involucrados. El que enseña, en la medida en que se consideran los procesos de enseñanza de los sujetos encargados de impartir educación, los procesos evaluativos utilizados por este y las diferencias individuales que lo atañen, es decir, su personalidad, sus creencias, valores, niveles de relación y su estilo cognitivo. Asimismo, la psicología educativa tiene en cuenta lo que los sujetos aprenden y lo que los educadores enseñan, dado que se necesita delimitar los contenidos del aprendizaje, realizar diferencias entre el diseño instruccional, como aquella toma de decisiones sobre la selección de contenidos de enseñanza, los objetivos, la metodología y el tipo de evaluación a utilizar, y el diseño curricular, entendido este como la concreción de contenidos y de intencionalidades educativas a desarrollar en un ámbito y nivel determinado de enseñanza. Por último, se hace necesario tomar en consideración el medio que involucra, tanto al sujeto que aprende como a aquel que imparte pautas de aprendizaje, es decir, existe amplia curiosidad en la actualidad por delimitar no solo las condiciones físicas de la institución educativa, sino también sus aulas, la sociedad inmediata que la rodea, el barrio, el tipo de familias inscritas en ella y aquellos aspectos que podrían denominarse de riesgo y/o protectores en el ambiente. En esta medida, puede decirse que la psicología no sólo se ha preocupado por los sujetos del contexto educativo, sino, también, por los sujetos en interacción con el contexto educativo y social.

Asimismo, y con el fin de ampliar las estrategias no sólo de intervención sino de potenciación de habilidades en los docentes, discentes y el contexto que los envuelve, existen otras tendencias de la psicología. En este sentido, cabe mencionar a Clark, citado por Smeby (1996), quien enfatiza en abordar diversos aspectos tales como: destrezas para tomar decisiones en el aula de clase, independiente de las personas que en ella se encuentren y su nivel educativo; conocimiento de los problemas especiales que enfrentan los estudiantes pertenecientes a minorías o que se presume en ellos retardo en el desarrollo; abordaje de factores que pueden influir en el desempeño, tal como el desarrollo cognitivo, la disciplina, los modos de evaluación entre otros; y la necesidad de capacitar a los maestros en destrezas que involucren el abordaje de situaciones del "mundo real", es decir, de las dificultades del contexto y de cómo el estudiante se involucra y vive en él. 
Basados en los planteamientos anteriores, puede decirse que esta disciplina psicológica se interesa por los procesos de cambio comportamental provocados o inducidos en las personas como resultado de su participación en actividades educativas. Se enfoca en la naturaleza de esos procesos de cambio, las características, los factores que los facilitan, los obstaculizan, la dirección que toman y los resultados a los cuales llegan. Dichos procesos son procesos de adquisición, es decir, dan lugar a un aprendizaje; son intencionales y finalizados, o lo que es lo mismo, responden a unas intenciones u objetivos educativos. Tienen lugar durante un período de tiempo relativamente largo, provocan efectos durables en las personas e implican reestructuraciones importantes del comportamiento.

Se esbozan así, algunos aspectos finales. En primer lugar, a través del creciente interés por la investigación y por aplicar los resultados de esta al desarrollo de un programa educativo integral, la mirada de los psicólogos se hace indispensable en la medida en que se intenta que los planes, los objetivos, el material pedagógico y la forma de evaluación se adecuen, no solo al proyecto curricular, sino que también se piensa el cómo diseñar estrategias que permitan al estudiante alcanzar las metas allí señaladas. A su vez, sobre la base de los hallazgos empíricos, los psicólogos educativos han creado programas de formación permanente del profesorado para mejorar el aprendizaje de asignaturas como lectura, escritura o matemáticas, de manera que aumenten la capacidad de aprendizaje de sus alumnos; es decir, se han estructurado sistemas de instrucción que permitan a la mayoría de los alumnos, a través de los procesos relacionales y de aprendizaje impartidos por los docentes, alcanzar notable éxito académico. Adicional a ello, los psicólogos educativos se interesan cada vez más en cómo la gente recibe, interpreta, codifica, almacena y recupera la información aprendida. Por tanto, la comprensión de estos procesos cognitivos ha permitido que se aporten estrategias fundamentales en el transcurso de resolución de problemas, el incremento de la capacidad memorística y los inicios no solo de un pensamiento creativo sino también el impulso para la creación de un producto creativo final. Finalmente, la reciente legislación que aboga por la inclusión de personas en situación de discapacidad o con dificultades emocionales y de aprendizaje en el contexto educativo, ha extendido el campo de la investigación, conllevando a la aparición de nuevas y mejores formas de evaluación, no solo de dichas discapacidades sino del hallazgo 
de necesidades individuales de los sujetos, con el fin de implementar competencias, tanto del docente para con ellos como de la institución misma.

Teniendo en cuenta lo anterior, no podría decirse que se han alcanzado todas y cada una de las pretensiones que demanda el mundo educativo. Sin embargo, el psicólogo se ha concientizado de que, dado que los procesos en este ámbito son tan complejos, puesto que se cuentan con numerosos factores y variables asociadas, se requiere una mirada multidisciplinaria, y, dentro de esta mirada, la de la psicología de la educación sigue siendo muy importante. Es decir, se necesita generar conocimientos, modelos explicativos e instrumentos de intervención, para orientar, guiar y explicar cómo los sujetos construyen el sentido, cómo las personas atribuyen el sentido de la actividad y la acción, cómo se comportan y qué factores están asociados a esto.

Todo esto es imposible que se pueda hacer al margen del contexto, de las condiciones, de las situaciones concretas en las que ese comportamiento, esa actividad realmente se despliega, es decir, el comportamiento humano no se puede entender al margen del contexto en el que se genera, y, por ende, uno de los grandes retos del psicólogo en la actualidad es, no sólo conocer el contexto sino interpretarlo, darle un significado y trabajar en pro de los sujetos vinculados en él, a través de él mismo.

\section{Sujetos inmersos en el contexto educativo}

Comprender las condiciones o diferencias individuales de los sujetos inmersos en el contexto educativo, requiere realizar una aproximación reflexiva de relación entre los diferentes conceptos que inciden en el desarrollo del ser humano. Es así como se plantea, en primera instancia, la necesidad de describir algunas posturas descriptivas sobre los sujetos y cómo estos actúan y toman un lugar en el mundo permeados por los contextos en los que se desenvuelven. Y, más adelante, la posibilidad de abordar con mayor profundidad las definiciones conceptuales de los términos diferencias individuales y riesgo, a partir de las posturas de diferentes investigadores que se han cuestionado sobre este asunto.

De manera inicial, se hace entonces menester mencionar dos reflexiones iniciales: Primero, la comprensión del contexto como una 
construcción humana, lo que hace imposible definir a los contextos independientemente de las diferencias que entre los mismos sujetos coexisten. En este sentido, Barker (1987) considera que el contexto en el cual un sujeto se desenvuelve es algo más que el espacio en el que este transita. Por tanto, este es interdependiente de los cambios individuales de cada uno de los sujetos que en él se circunscriben; los cambios de un sujeto en contexto afectan necesariamente a aquellos que acompañan a este y por ende el contexto se transforma. Por otro lado, se considera que el contexto es un constructo eminentemente social, dirigido y organizado por reglas humanas; por tanto, los propósitos y los objetivos de las personas que forman los contextos pueden hacer variar su organización, es decir, las personas contribuyen en la creación de la dinámica de su contexto, pero dicha dinámica se consolida como un factor influyente en la perspectiva de desarrollo del ser humano. Al respecto, Cohen y Siegel (1991) advierten que los contextos rodean al comportamiento humano, lo transforman a través de su influencia y los sucesos vivenciados en él.

Ahora bien, pensar en la existencia de una relación estrecha entre las diferencias de cada individuo y su contexto, no puede verse como una directa influencia de variables, sino que lo que se debe pretender a través del acercamiento teórico, investigativo y de intervención, es enfatizar cómo ambas al implicarse remiten a pensar en las deficiencias y potencialidades que el ser humano pone en marcha en el momento de actuar ante el mundo.

Y es aquí donde tiene lugar pensar la psicología diferencial, como una disciplina entregada a la exploración básica de las diferenciasindividuales que, si bien inicia con el objetivo de establecer las estructuras correspondientes a la organización de constructos psicológicos, tales como la inteligencia o la personalidad de los individuos, al amparo de una tecnología psicométrica como estructuras que articulan las dimensiones básicas que constituyen dichos constructos, este aspecto ha sido ampliamente cuestionado por su carácter descriptivo, de clasificación de los individuos y sin intención de explicar los comportamientos y lo propio de las diferencias individuales. Todo ello genera una visión estática de la psicología, que no permite acceder a un estudio integral del sujeto mismo, de este en su contexto y el cómo este produce efectos en él. En últimas, no se logra comprender el cómo se originan, mantienen y exacerban las diferencias individuales manifiestas en los sujetos. 
Respecto al nacimiento de esta disciplina, Dubois (1970) afirma que el origen de la evaluación de las diferencias individuales en determinadas aptitudes apunta hacia China, puesto que allí se utilizaba un sistema de exámenes que medía las capacidades individuales que servían de selección a los más fuertes. En este sentido el abordar dicho concepto ha estado históricamente asociado a la evaluación, selección e implementación de los "más aptos".

Por otro lado, se encuentra a Hipócrates (460-377 A.C.) aproximándose a una idea sobre diferencias individuales cuando afirma que el hombre es imagen y semejanza de la naturaleza y sus cuatro elementos; dichos elementos son diferencias constitutivas del ser humano y poseen una característica esencial: la estabilidad. Posteriormente, Galeno (129-199 A.C.) ampliaría el número de tipos humanos a nueve, teniendo en cuenta que la combinación entre estos es la que da lugar a diferencias individuales en la manifestación comportamental y, ¿por qué no?, de la personalidad de los diferentes sujetos.

En este sentido, Eysenck (1989) menciona que algunos de los principales conceptos sobre los estudios en el área de personalidad han estado influidos por términos como rasgo, tipo, constitución y factores ambientales, los cuales han girado no sólo en determinar los tipos de personalidad, sino cómo influyen estos en las diferencias individuales. Por su lado, Robach (1931), comenta que la virtud de las anteriores contribuciones estriba en que posibilitan afirmar, sin ningún género de dudas, el carácter universal y atemporal de las diferencias individuales humanas, así como de las diversas problemáticas que la existencia de las mismas ha sugerido desde la antigüedad. Forteza (1993), de forma muy concisa, señala que el estudio de la variación individual consiste en describir, clasificar, ordenar y, hasta cierto punto, explicar las divergencias comportamentales esencialmente interindividuales, pero también intraindividuales e intergrupales.

En la actualidad, y partiendo de nuevos lineamientos investigativos, tales como la metodología correlacional y el análisis factorial en particular, y, a su vez, la inclusión de otras disciplinas científicas, se pretende establecer las estructuras correspondientes a la organización de constructos psicológicos relevantes tales como la inteligencia, la personalidad, el desempeño cognitivo, etc. 
Al respecto, esta disciplina psicológica posee, como temas de interés, causas genéticas y ambientales, efectos de interacción, temperamento, inteligencia, carácter, actitudes, capacidades, entre muchos otros. De acuerdo con Espinosa, Colom y Quiroga (1997), las investigaciones de diferencias individuales en las capacidades cognitivas tradicionalmente estudiadas por la psicología experimental general se han dado en los campos relacionados con el estudio de la percepción (Tzeng \& Hung, 1985); estudio de la memoria (Underwood, 1975; Dempster, 1981; Gitomer \& Pellegrino, 1985); relación de problemas (Simon \& Sumon, 1978); razonamiento humano (Egan \& Grimes-Farrow, 1982); el estudio de la capacidad espacial (Cooper \& Mumaw, 1985); imágenes mentales (Poltrock \& Brown, 1984), (Kosslyn, 1984); el estudio de las habilidades cognitivas complejas (Egan \& Gómez, 1985); la atención (Lansman, Poltrock, \& Hunt, 1983); entre otras representaciones cognitivas. Por su parte, desde el enfoque diferencial psicométrico, se han estudiado temas como personalidad e inteligencia (Cattel, 1890; Spearman, 1927; Binet \& Simon, 1905; Eysenck, 1967; Guilford, 1967; Royce \& Powell, 1983).

En este sentido, en el que los grandes temas desde el enfoque del procesamiento de la información son, de acuerdo con Sternberg (1985), los procesos mentales que constituyen el rendimiento inteligente en diferentes tareas, la rapidez y exactitud con la que se ejecutan estos procesos, en qué estrategias se combinan estos procesos mentales para la resolución de tareas, en qué formas de representación mental actúan estos procesos y estrategias, y cuál es la base cognitiva organizada de estas formas de representación, cómo afecta y cómo se ve afectada por los procesos, estrategias y representaciones que utilizan las personas.

El estudio de las diferencias individuales viene así determinado, básicamente, por la búsqueda de unidades significativas de diferenciación psicológica entre los individuos. La posible relevancia de dicha variabilidad no estriba, meramente, en su existencia. Las dimensiones a lo largo de las cuales los individuos difieren deben cumplir unos requisitos fundamentales, relativos a su estabilidad temporal y consistencia transituacional en la conducta individual, para poder ser establecidas como categorías significativas de diferenciación y no meros efectos espurios, tal y como son entendidos desde una psicología de corte generalista. Es así como, de acuerdo con Krahé (1992), citado por Sánchez y Paniagua (1996), el concepto de 
consistencia es central al estudio de las diferencias individuales, lo que implica que, para detectar las características que distinguen al individuo de los demás, deben buscarse diferencias consistentes entre los individuos a través de las situaciones y del tiempo. Además, para demostrar la estabilidad y permanencia se requiere evidencia de consistencia intraindividual, también temporal y transituacional. Y, por último, para poder explicar la conducta de un individuo como fruto de la manifestación de alguna disposición interna, es esencial probar que esta modela la conducta de forma consistente y fiable en diferentes situaciones.

Asimismo, se entiende que el remitirse a pensar estas variables, implica tener una óptica integral, en la medida en que obliga a no quedarse con un análisis tradicional de aquellos sucesos no gratificantes e indeseados para el sujeto o su comunidad, sino comenzar a adoptar una postura que podría denominarse ecológica, en la medida en que remite a pensar la situación de riesgo a través del cómo el ser humano la vive y se afecta, desde la sensibilidad del propio sujeto, el momento histórico, el contexto y las decisiones político sociales que a este afectan.

Por todo lo mencionado con anterioridad, la psicología de las diferencias individuales se caracteriza por tener un acercamiento nombrado como nomotético, es decir, la individualidad es concebida como una agrupación de características globales de la población de la que el individuo es representativo. Y, en consecuencia, el estudio de dichas personas, posibilita la comparación, análisis y discusión de aquellos aspectos diferenciales que constituyen herramientas específicas para diseños posteriores de intervención y mayor nivel de evaluación de la población.

Al plantearse, en la psicología diferencial, que cada persona es un hecho único y diverso, y que, consecuentemente, no es posible postular la existencia de leyes generales que puedan aplicarse por igual a todos los individuos, se han generado cuestionamientos, como ya se mencionó, en varios campos de la psicología. Uno de ellos es el de la educación, donde existen razones de distinta índole para identificar y medir las diferencias individuales de las personas, así: pueden describirse diferencias entre los individuos para identificar a personas que poseen una determinada característica o que la poseen en cierto grado, con la finalidad de otorgar premios, o simplemente escogerlas por motivos diversos, también pueden formular predicciones respecto al comportamiento futuro de las personas 
para seleccionarlas educativamente, ya sea identificar el perfil de las características para situarlo correctamente respecto a una situación o tarea o diagnosticar las dificultades que presenta una persona a fin de prescribir el tratamiento más adecuado.

Las diferencias individuales han sido definidas, en términos de tipos, rasgos, destrezas, ejecuciones en test psicométricos y aptitudes, que se constituyen como características estables, altamente sensibles e influenciables por el medio sociocultural o como resultado de la interacción con las características del medio exterior. En el campo educativo, se ha considerado como más relevante el constructo de aptitud; por esta razón el estudio y explicación de los cambios en el comportamiento humano, acapararon la atención de los investigadores interesados en evaluar las aptitudes individuales relacionadas con el aprendizaje escolar con el fin de elaborar teorías y propuestas de intervención tendientes a mejorar la influencia de las prácticas educativas en los procesos de desarrollo personal, con base en la supuesta relación entre medidas de las diferencias individuales y variables del aprendizaje. Las aptitudes entendidas como indicadoras del potencial cognitivo individual, con respecto a la adquisición de nuevos conocimientos, se configuraron como un constructo aceptado y válido por su utilidad en relación al diseño de las prácticas educativas y al diagnóstico de las dificultades de aprendizaje.

En esta línea, Pellegrino (1996), define las aptitudes como constructos psicológicos acerca de las diferencias individuales en situaciones de enseñanza aprendizaje (naturaleza predictiva de las aptitudes para aprender en situaciones instruccionales específicas), este planteamiento lleva a la necesidad de que las personas sean objeto de tratamientos educativos diversos en función de sus diferencias de aptitudes. Snow (1986) considera todos los constructos sobre las diferencias individuales, relevantes para la educación en términos de aptitudes, para significar que los aspectos del estado actual de una persona son propedéuticos, una palabra de raíz griega que significa necesario para la preparación de cara a un logro futuro. En este sentido, la educación es un programa de desarrollo de aptitudes en el sentido de que su principal objetivo es preparar al ser humano para posteriores etapas de la vida. Por lo tanto, las mejoras educativas más importantes son las que hacen que la educación sea adaptativa a las diferencias aptitudinales 
al inicio de la instrucción y fomentan el desarrollo de las aptitudes durante ella y más allá.

Es necesario tener claro que las aptitudes no se encuentran simplemente en el interior del individuo como listas de entidades fijas e independientes, y siempre vigentes; más bien actúan concertadas ante una demanda situacional, es decir, muestran combinación compleja en la interacción persona-situación, dando como resultado fuerzas o debilidades relativas a condiciones ambientales particulares (Escoriza, 1998).

Por otro lado, el estudio de las diferencias individuales no puede considerarse un objetivo en sí mismo, sino en la medida en que estas constituyen un factor primordial a tener en cuenta en el diseño de propuestas educativas. Por esto, una de las cuestiones centrales a las que se enfrenta la psicología de la educación es la problemática de la adecuación de la enseñanza a las características diferenciales que presentan los alumnos. Parafraseando a Miras y Onrubia (1996), enseñar de manera eficaz es en realidad enseñar de manera adaptada a las características de los alumnos, y el problema de respuesta a la diversidad, es, en definitiva, el problema mismo de los procesos de enseñanza.

Otro aspecto importante a resaltar desde este nuevo enfoque, es el hecho de que asumir que las personas son diferentes, no implica aceptar que las diferencias son atribuibles a déficits o discapacidades (sensoriales, cognitivas, psicomotoras, etc.) sino que deben ser interpretadas en términos de funcionamientos psicológicos diferentes (diversidad de capacidades, intereses, ritmos de aprendizaje, etc.) que traducen necesidades educativas personales a los que las situaciones de enseñanza-aprendizaje deben proporcionar respuestas educativas diversificadas y adaptadas a dichas necesidades (atención educativa a la diversidad). Puesto que la diversidad es un hecho inherente al desarrollo humano, la educación escolar tendrá que asegurar un equilibrio entre la necesaria comprensividad del currículo y la innegable diversidad de los educandos. La psicología de la educación, en bucle con la psicología de las diferencias individuales, deberá preocuparse por responder a los interrogantes que se interesan por conocer los ámbitos en los que se genera la diversidad y cómo se pueden elaborar respuestas educativas que tengan en cuenta dicha diversidad. 
De acuerdo con Lluis (2004), el trabajo del psicólogo escolar en el campo de las diferencias individuales ha de servir, entre otros objetivos, para identificar a los alumnos con unas determinadas necesidades educativas, para promover adaptaciones curriculares, para aconsejar itinerarios educativos alternativos, para orientar profesionalmente y para guiar cualquier tipo de intervención, ya sea efectuada por los propios educadores del centro o ya por personal especializado, externo o contratado.

Existen diferentes temas de interés investigativo desde esta perspectiva, entre los cuales están la consideración de los componentes motivacionales y cognitivos en el diseño y desarrollo de procesos instruccionales adaptativos, el análisis de los estilos o estrategias de enseñanza y aprendizaje, la explicación dinámica de las diferencias individuales en cuanto a procesos, estrategias y conocimientos en contraposición a una explicación estadística de los constructos hipotetizados, la aplicación de formas de evaluación dirigidas a obtener información con respecto a la distinción de los niveles de ejecución individual o con apoyo de un experto.

Puede observarse, entonces, cómo la exploración básica de las diferencias humanas en el ámbito educativo, se convierte en un medio para generar teorías sólidas que ayudan a plantear preguntas relevantes y a interpretar las respuestas que se van encontrando en distintas situaciones básicas y aplicadas.

Por su parte, al analizar cuestiones tales como la distinción entre rasgo y estado, o la elaboración de constructos cognitivo-conductuales de diversa índole, expectativas generalizadas de control, o estilos atribucionales, estas se constituyen en nuevas unidades de análisis de diferenciación individual desde una perspectiva funcional o procesual. En otro tipo de aproximaciones más cercanas a un entendimiento más cualitativo de la individualidad, se propone el estudio de las estrategias diferenciales que pueden caracterizar a los individuos, en el afrontamiento de las situaciones que son definidas como variables personales internamente coherentes e impulsoras de una dirección específica en la interacción del individuo con su entorno. No se pretende abandonar el estudio de posibles estructuras psicológicas estables, sino que se aboga por su complemento con un análisis posterior de procesos, siempre entendidos desde el marco de procesos diferenciales, es decir, de diferencias individuales en procesos psicológicos. Un ejemplo de las nuevas aportaciones, que el estudio de las diferencias individuales 
puede realizar desde una perspectiva transaccional, en donde estructura y procesos parecen conjugarse convenientemente, es el área de la psicología de la salud.

En consecuencia, Sánchez y Paniagua (1996), plantean que ha llegado el momento de que la psicología de las diferencias individuales sea capaz, no de estudiar al individuo y a la situación, sino al "individuo en situación", y ello implicará, necesariamente, un menor nivel de generalización de los resultados de los diseños, pero, indudablemente, una mayor proximidad a la realidad contextualizada, en donde la conducta tiene un lugar y un conocimiento más preciso del carácter funcional de las diferencias individuales.

Puede denotarse, entonces, que el estudio de las diferencias individuales, históricamente, surge como planteamiento alternativo a un enfoque generalista centrado en la definición y el análisis funcional de la conducta humana en cuanto al hombre en sociedad. Por tanto, la existencia de este estudio, lleva a la detección de fuentes de variación particular que permite distinguir a aquellos individuos en sociedad, unos de otros. Adicional a ello, el estudio de las diferencias individuales ha estado vinculado a una concepción internalista de las mismas de forma sistemática, es decir, más centrado en la relevancia de la persona que en la situación manifiesta en la conducta, tal como se deriva de los postulados principales de los modelos de rasgos. En otras palabras, aquellas características que diferencian a los individuos responden a cualidades personales preexistentes que "se ubican en el interior" de las personas y dan cuenta de su comportamiento a lo largo del tiempo y de las distintas situaciones; es decir, son determinantes potenciales de la conducta, y, por añadidura, de una conducta diferenciada.

En este punto, es necesario señalar que, si bien el abordaje de las diferencias individuales remite a pensar las particularidades de los individuos, cabe mencionar otro factor que está estrechamente ligado al individuo mismo, es decir, la inclusión de este en sociedad. Cabe aclararse que no se pretende, necesariamente, hacer referencia a un contexto que proporciona seguridad suficiente y a la vez que garantice la potencialización de habilidades individuales, sino que se señala un contexto que puede nombrarse como aquel donde el sujeto en interacción se desarrolla a partir, en relación y bajo situaciones sociales de riesgo. 
Es así como, al preguntarse por estas características individuales y su despliegue en un contexto cargado con particularidades específicas en lo que concierne a su desarrollo, surge, en los últimos años, una tendencia a emplear la noción de riesgo, siendo nombrada con alta frecuencia dadas las condiciones encontradas en el medio, que son percibidas por los diferentes sujetos como problemáticas en la medida en que generan disfunción de diversa índole en los seres humanos. Sin embargo, su definición suele ser dificultosa dado que se encuentran diversas posturas; una de ellas es la proporcionada por Castel (1981) quien define riesgo como la relación de datos generales impersonales o factores que hacen más o menos probable el acontecer de conductas indeseables.

En este mismo sentido, Casas (1994) propone que factor de riesgo es un concepto referido a determinadas condiciones biológicas, psicológicas o sociales medidas por variables directas, que actúan probabilísticamente en las situaciones asociadas o implicadas con emergencias sociales. Este mismo autor, años más tarde, presenta una propuesta conceptual diferente, donde postula que, más que hablar de situación o factor de riesgo, se debe pensar en el uso del neologismo disadaptación, dado que en sí mismo es un fenómeno psicosocial que emerge de la relación conflictualizada para con determinadas conductas individuales o grupales que mantiene una comunidad o sociedad, así como el clima social que se construye alrededor de tales conductas en un período histórico concreto.

De acuerdo con Radke y Sherman (1992), la población infantil, específicamente, muestra durante su desarrollo una contradicción. Esta se produce, dado que estos son inherentemente vulnerables, a la vez que muestran una fuerte determinación por sobrevivir y crecer. Esta característica propia de los niños, ha dado origen a múltiples estudios sobre qué es aquello que hace posible que estos se desarrollen adecuadamente, tanto física como psicológicamente, bajo condiciones que atentan contra su sobrevivencia o bienestar.

Las autoras mencionadas señalan que la importancia que se asigna a las investigaciones sobre el riesgo y sus efectos, data de diversos estudios desde 1946 (Spitz \& Wolf, 1946, citados por Radke \& Sherman, 1992), donde se encontró que niños institucionalizados con características de maltrato y/o niños con falta de una figura significativa cercana, sufrían de depresión, y que incluso morían. Asimismo, (Harlow, 1958, citado 
en Radke y Sherman, 1992), denota cómo, a partir de un estudio similar con animales, se observa que las condiciones ambientales severamente deprivadoras, tienen un significativo impacto en el desarrollo tanto social, emocional, cognitivo como físico de los seres humanos, a la vez que en el grado de bienestar alcanzado por estos.

En Giddens (1990) la noción de riesgo se asocia con la palabra inglesa, deletreada para su versión en francés como risque, misma que se continuó utilizando junto con la nueva palabra anglosajona risk, que sería destinada para su uso en el campo de los seguros.

Douglas y Wildavsky (1982), reconocidos por sus trabajos antropológicos sobre riesgo y cultura, han observado que la connotación de la palabra riesgo ha ido cambiando a través del tiempo. De manera inicial, fue introducida en el siglo XVII, en el contexto del juego, connotando probabilidad de que un hecho ocurra, combinado con la magnitud de pérdidas y ganancias. En la actualidad, riesgo tiende a estar como concepto asociado a desenlaces negativos, en especial en el plano profesional o técnico del término. Douglas y Wildavsky (1982), remitiéndose a La Royal Society, definen riesgo como "la probabilidad de que ocurra un hecho particular adverso, durante un período de tiempo dado".

El riesgo, pensado desde la teoría sociológica y enmarcado en la sociedad contemporánea y sus nuevas formas de vida social, se encuentra conceptualizado en Giddens (1990) como "aquellos modos de vida u organización social que surgieron en Europa desde alrededor del siglo XVII en adelante y cuya influencia, posteriormente, los ha convertido en más o menos mundiales". (p.57)

Por su parte Beck (1986, 1996), basado en el enfoque de la "Sociedad del Riesgo", resalta que el desarrollo científico y tecnológico trae consigo mayores niveles de inseguridad caracterizadas por ser indiscriminadas y globales. Por tanto, calcular, gestionar y evitar el riesgo es cada vez más difícil; y, como ejemplo de ello, está el que instituciones como el Gobierno o la Industria se vean cada vez más amenazados y las sociedades que estos permean demanden más seguridad y calidad de vida. Asunto que conlleva a que las personas se vean abocadas a decidir sobre su propia seguridad.

Si bien autores como Beck (1996) y Giddens (1990) comparten puntos en común en torno al análisis del riesgo, al entenderlo como un 
elemento característico de la modernidad fundamentado en la radicalización y universalización, la diferencia básica entre ellos está dada en que Beck se concentra preferentemente en los problemas institucionales planteados por la modernidad reflexiva y Giddens se emplaza más en los conflictos de la identidad individual y el desarrollo del sujeto reflexivo.

Beck (1996) señala como aspectos de la sociedad moderna la producción de riesgos políticos, ecológicos e individuales, los cuales cada vez se encuentran más por fuera del control de las instituciones encargadas de garantizar la seguridad de la sociedad, al tiempo que precisa que, como particularidades de las sociedades del riesgo, se encuentra la insondable ruptura entre las sociedades industriales y las sociedades de riesgo, donde la primera pasa por la sustitución del orden, la jerarquía y la autoridad, hacia la incertidumbre y ambivalencia que identifica la sociedad del riesgo.

Para los autores anteriormente mencionados, los cambios entre lo que se ha denominado modernidad simple — centrada en los cambios sociales en la racionalidad teleológica- - y la modernidad reflexiva — la cual piensa la sustitución de los modelos de racionalización y modernización por conceptos como auto-transformación, auto-amenaza y auto-destrucción-, son asuntos que conllevan a crisis de la racionalidad y a nuevas contradicciones y problemas sociales, donde entran en contradicción las grandes teorías de grupos y las teorías de la individualización.

\section{Necesidades del contexto escolar y vulnerabilidad social}

Hastaahora, es clarocómolosprofundoscambiosquehaexperimentado la psicología educativa durante las últimas décadas, han conllevado a que los estudios alrededor de ella tengan como característica importante el abordar el contexto, entendiendo a este como todos y cada uno de los ambientes, con sus características físicas y sociales, que enmarcan y rodean el cambio comportamental humano (Clemente y Hernández, 1996). Las investigaciones, en psicología educativa, han acumulado una base o cuerpo de conocimientos que incluyen factores como las influencias ambientales y culturales sobre el aprendiz, el funcionamiento cognoscitivo de los estudiantes, el manejo del salón de clases, la forma en que los estudiantes aprenden y la manera en que esas variables se relacionan con los docentes 
y con la enseñanza. Enseguida se esbozan los aspectos metodológicos sobre los cuales se han llevado a cabo las diferentes investigaciones desde esta disciplina.

Por esta razón, es necesario mencionar que no es desconocido, para el mundo y para la sociedad colombiana en particular, que Colombia presenta serias dificultades en el ámbito social, económico, familiar y político. Cada vez es más evidente reconocer la inexistencia de políticas educativas en relación con aquellos niños que están inmersos en el conflicto y para quienes no se logran establecer diferencias en torno a estilos de aprendizaje y comprensión de su aprovechamiento escolar, y pocas son las investigaciones que denotan las estrategias de socialización y convivencia en este contexto.

Partiendo de esta concepción, se han motivado durante los últimos años diversas investigaciones en el campo escolar, dado que implica no solo tener en cuenta factores individuales, sino también sociales y familiares, que comprometen la vida académica de los niños. Es así como Hederich y Camargo (1999) indagaron acerca de los estilos cognitivos y el logro académico de niños que se encuentran cursando los grados de básica y media de educación, teniendo en cuenta factores psicosociales influyentes en estas, como el nivel socioeconómico, el nivel sociocultural, la conformación del grupo familiar y la proveniencia ecocultural de la familia. De acuerdo con los resultados obtenidos, se describe que un primer grupo de alumnos tiene como característica un estilo cognitivo sensible al medio en el que se desenvuelve, alta repitencia escolar, ausencia de medios tecnológicos en casa y en el aula, extra edad en los cursos, actitud negativa a las materias y co-residencia de familias extensas. El segundo grupo, obtuvo bajas competencias en las pruebas, pero alto rendimiento según los maestros, presentan características similares a los anteriores, pero con altos niveles de disciplina escolar y bajos niveles de movilidad escolar. El tercer grupo, obtuvo altas competencias en las pruebas, pero bajo rendimiento según los maestros, presentando como características un estilo cognitivo independiente, niveles incipientes de ausentismo escolar y niveles relativos de movilidad. Por último, se describe un cuarto grupo que presenta alta competencia y rendimiento escolar, y sus características son estilo cognitivo independiente del medio, actitudes y vivencias positivas en el medio escolar 
y social, situación de adelanto con respecto a la edad promedio escolar, medios tecnológicos en casa y niveles educativos altos en padres y madres.

Santostefano, Quiroga y Rooney (2001), a partir de la teoría de los controles cognitivos, exploraron cómo se relaciona el funcionamiento cognitivo de los niños con los factores estresantes reportados por ellos, tales como disparos, observación de robos, muerte y violencia intrafamiliar. Lo anterior da como resultado que los niños que informan haberse sentido afectados por discusiones y gestos amenazantes entre adultos, especialmente familiares, cometen más errores mientras centran la atención ante estímulos escolares. Por otro lado, los niños que informan haberse sentido afectados por disparos y peleas, tuvieron más dificultad en lo concerniente a la recuperación de la información y los niños que reportan otro tipo de factores estresantes, como muerte de un vecino significativo o familiar, presentan bajo rendimiento en la evocación, atención y alta percepción de vulnerabilidad al daño.

Igualmente, en el tema del alto riesgo, Dubowitz, Papas, Black y Starr (2002), realizaron una investigación que tuvo en cuenta variables criterio como los factores de riesgo sociodemográficos, depresión materna, riesgo medio ambiental, riesgo físico y psicológico, barrio y nutrición. Este estudio encontró como resultados principales la existencia de una relación directa entre el contexto de pobreza y el bajo desarrollo cognitivo de los niños y, a su vez, se observó que dicho resultado se correlaciona con el bajo nivel de afrontamiento de los padres y la resolución agresiva de conflictos de estos. Asimismo, la evaluación de aquellos niños que reportaron experiencias de abandono o violencia intrafamiliar demuestra que presentan mayores problemas psicológicos y del comportamiento. Sin embargo, en los niños con edades entre 3 y 5 años no se encuentra que las experiencias múltiples de abandono o agresión se correlacionen con desarrollo de conductas desadaptativas.

En este sentido, mucho es lo que desde la investigación psicoeducativa se piensa y se expresa en contundentes discursos con respecto a los factores involucrados en el aprovechamiento escolar de los niños; se mencionan factores como la violencia, fenómeno que irrumpe a diario en las escuelas, pero sobre el que no siempre se sabe muy bien qué hacer cuando adquiere entidad de realidad. A su vez, se piensa en la falta de equidad social como alto factor contribuyente para las dificultades en el desempeño de los niños 
en su actividad cognitiva y, sobre todo, se examina en la actualidad lo favorable o desfavorable que puede resultar el entorno social en el cual el niño se desenvuelve y, a su vez, asimila información.

Ante esto, según Ross, Marshall y Scott (2001), los niños de zonas periféricas que, a su vez, en diversas ocasiones, se equiparan indiscriminadamente como delincuentes, presentan como características específicas, alta impulsividad, pensamiento concreto, rigidez cognitiva, resolución inadecuada de problemas, inadecuada aplicación de los valores y de razonamiento crítico; pero, a la vez, alta capacidad de adaptabilidad al entorno, es decir, cada vez más se observa como factor importante la urgencia de ser estudiados los factores resilientes en los niños.

Es conocido que la gravedad de la violencia intrafamiliar en Colombia está en que condiciones como el maltrato al menor, el abuso de drogas, la explotación, violación a los menores y la conformación de grupos ilegales, en su mayoría por niñas y niños víctimas del conflicto, se han convertido en cotidianidad y se constituye casi en un medio de sobrevivencia. En este mismo sentido, el abuso infantil ha existido siempre, aunque ha sido durante los últimos 150 años cuando ha ido emergiendo como un problema social y una considerable cantidad de instituciones sociales y legales se han ocupado de este (Cortés \& Cantón, 1997).

Por otro lado, el desplazamiento en Colombia es "La otra guerra detrás de la guerra". Se estima que Colombia ocupa el segundo lugar en relación con el número de desplazados, en el mundo (por lo menos 2.5 millones), involucrando en ello a la población infantil, dado que los niños y los adolescentes son quienes conforman la gran mayoría de población desplazada y maltratada a nivel social, emocional y educativamente (CODHES, 1999). De este mismo modo, es necesario tener en cuenta que el impacto del conflicto en los niños colombianos varía entre los diversos segmentos de la población, tales como aquellos que viven en diferentes regiones, niños campesinos, indígenas y afro colombianos, que no tienen acceso a educación, salud y/o a un hogar estable, es decir, aquellos que han sufrido el desplazamiento.

Por otro lado, las cifras de niños víctimas de abuso sexual se incrementa cada día. Se vive en un ambiente social de agresión generalizada y de maltrato y abuso sexual de los niños; y esto no es difícil de corroborar puesto que, a 
diario, los casos reportados van en aumento y los estudios internacionales así lo corroboran (Ministerio de Salud de Chile, 1998). Al hablar de abuso sexual, se remite necesariamente a tener en cuenta los diferentes continentes, dado que se constituye en una problemática mundial. Es bien sabido que, en diversos países del continente suramericano, como Bolivia, Ecuador, Brasil etc., el aumento de víctimas infantiles es cada vez más creciente, al igual que en otros lugares del mundo, como España, Francia, Alemania e incluso África (López, Carpintero, \& Hernández, 2002). Sobre la violencia infantil, es conocido que esta afecta el capital humano, es decir, reduce los logros educativos en términos tanto de años de escolaridad como de aprovechamiento académico. En esta investigación se muestra cómo el enfrentar situaciones sociales y familiares negativas, generan vicisitudes como bajos logros, inadecuada percepción de logro académico por parte de los estudiantes, docentes y padres de familia, inasistencia escolar y déficit en el grado de avance académico.

En otra vía de análisis, pero involucrando factores relacionados con lo anterior, vale la pena discutir que, si bien los niños en alto riesgo tienden a presentar diversos conflictos, propios del entorno en el que se encuentran, es decir, dificultades en el comportamiento, en la adaptación, y habilidades básicas de sobrevivencia, su percepción en torno al proceso de enseñanza es diferente al del logro escolar. Lo anterior, dado que dichos alumnos no muestran estrategias claras de planificación en el proceso de mejoramiento escolar y, por tanto, el proceso de enseñanza está fuertemente ligado a factores motivacionales por parte de los alumnos y las experiencias sociales y familiares de estos (Agudo \& Ortiz, 2003). En relación con esto, Hederich y Camargo (1999) comentan cómo los niños cognitivamente se tornan sensibles al medio en el que se desenvuelven, es decir, en los niños cuyo contexto se vislumbra como desfavorable se encuentra alta repitencia escolar, extra edad en los cursos y actitud negativa a las materias.

Una vez explorado el marco investigativo y el panorama contextual, y centrándose en la especificación conceptual de los términos que se avizoran en lo antes mencionado, Richters y Weintraub (1992) consideran importante distinguir entre lo que ellos denominan desadaptación y el concepto de vulnerabilidad. Estos autores argumentan que un comportamiento desadaptado no es sinónimo de ser vulnerable a algún desorden, sea este adquirido o heredado. Esta observación la hacen sosteniendo que la mayor 
parte de las consideraciones, respecto de la desadaptación que tienen ciertos comportamientos, se basan en evaluaciones de sujetos ajenos al propio individuo. Por ende, es así como, los sujetos que se apartan de algún modo del comportamiento que en términos estadísticos se denominan promedio respecto al grupo de referencia, son considerados desadaptativos.

Ahora bien, estos comportamientos delimitados como desadaptados, pueden resultar adaptativos a las características contextuales en las que se desenvuelve en un momento determinado el niño o adolescente. De acuerdo con Radke y Sherman (1992), el concepto de vulnerabilidad precisa de dos revisiones básicas: una pensada desde el concepto mismo y otra que implica el revisar el significado de riesgo y factores protectores asociados a este. En relación con el concepto teórico de vulnerabilidad se piensa, en primera instancia, en la asociación de este a niveles de estrés y su manejo inadecuado, es decir se le plantea como resultante y, por otro lado, el concepto de vulnerabilidad apunta a una dimensión continua del comportamiento que va desde la adaptación exitosa al estrés, a una que no lo involucra.

En relación con el significado de los conceptos de riesgo y factores protectores se cuestiona la universalidad de ellos en términos teóricos o si más bien deben estar ligados a las características de las personas. Lo anterior, dado que el significado sobre un fenómeno o concepto connota para diversas personas una relación diferente. En torno a lo anterior, Rutter (1987) señala que una misma variable puede actuar bajo distintas circunstancias, tanto en calidad de factor de riesgo como de protector. Y, por tanto, el significado que de ella se tenga actuará en consonancia.

\section{Conclusiones}

Bajo la lógica anteriormente descrita, el texto apunta a delimitar que, si bien la psicología diferencial ha centrado su interés en factores como la personalidad, la inteligencia y el papel de los padres, entre otros aspectos, la realidad actual le impone decididamente estudiar el contexto, dado que este se denota como fuente primordial, en tanto existe alta relación con él y el desarrollo de los seres humanos. 
En este sentido, el poner a dialogar tres grandes variables, como lo son las diferencias individuales, el concepto de riesgo y el de vulnerabilidad, remite a pensar en cómo estas se vislumbran en otros ambientes que, si bien hacen parte de la dinámica social, deben particularizarse para una mejor comprensión. Es el caso, entonces, de la escuela, el lugar de trabajo e incluso la familia.

\section{Referencias}

Agudo, I. M., \& Ortiz, M. (2003). Percepción del proceso Enseñanza Aprendizaje en alumnos de centros y contextos de alto Riesgo. Revista electrónica de investigación psicoeducativa y psicopedagógica, 1(2), 97 - 113. Recuperado de http://www.investigacion-psicopedagogica.org/revista/articulos/2/espannol/Art_2_10.pdf

Barker, R. (1987). Theoretical propositions of life -span developmental psychology: on the dynamics between growth and decline. Developmental Psychology, 23(5), 611 - 626. http://dx.doi. org/10.1037/0012-1649.23.5.611

Beck, U. (1986). La sociedad del riesgo. Hacia una nueva modernidad, Barcelona: Editorial Paidós.

Beck, U. (1996). La Sociedad de Riesgo. En J. Beriain (Comp.), Consecuencias Perversas de la Modernidad, Barcelona: Anthropos.

Beltrán, J. A. (1996). Concepto, desarrollo y tendencias actuales de la Psicología de la Instrucción. En J. Beltrán \& C. Genovard (Eds.), Psicología de la Instrucción (pp. 19-86). Madrid: Síntesis.

Beltrán, J. \& Bueno, J.A. (2004). Psicología de la educación: evolución histórica y concepto. En E. Gonzalez \& J. Bueno (Eds), Psicología de la educación y del desarrollo en la edad escolar. España: Editorial CCS

Bronfenbrenner, U. (1979). The experimental ecology of education. Educational Research, 5(9), 5-15. http://dx.doi.org/ 10.2307/1174755

Bueno, J. A. (1993). La motivación en los alumnos de bajo rendimiento académico: desarrollo y programas de intervención (Tesis doctoral). Universidad Complutense de Madrid: España. Recuperado de https://eprints.ucm.es/2237/1/T17692.pdf 
Bueno, J. A. (2004). Métodos de investigación en psicología de desarrollo y de la educación. En E. González, Psicología de la educación y del desarrollo en la edad escolar. España: Editorial CCS

Bueno, A. \& Castanedo, C. (1998). Psicología de la educación aplicada. Madrid: CCS.

Casas, F. (1994). Prevenció i qualitat de vida dels infants. Forum. Revista d'Informació y Investigació Socials, Desembre, 40-47.

Castel, R. (1981). La gestión de los riesgos. Barcelona: Anagrama.

Clemente R. A. \& Hernandez, C. (1996). Contextos de Desarrollo Psicológico y Educación. Archidona, Málaga : Aljibe.

Cohen, R., \& Siegel, A.W. (1991). Context and Development. Hillsdale, NJ: Lawrence Erlbaum Associates.

Coll, C., Palacio, J., \& Marchesi, A. (1990). Desarrollo psicológico y educación. Madrid: Alianza.

Consultoría para el Desplazamiento Forzado y los Derechos Humanos [CODHES] (1999). Desplazamiento y Violencia en una Nación Fragmentada: Colombia. Bogotá, Colombia: UNICEF.

Cortés, M. R., \& Cantón, J. (1997). Malos tratos y abuso infantil. Madrid: Siglo XXI de España Editores, S. A.

Dempster, F. N. (1981). Memory span: Sources of individual and developmental differences. Psychological Bulletin, 89(1), 63-100. http:// dx.doi.org/10.1037/0033-2909.89.1.63

Douglas, M., \& Wildavsky, A. (1982). Risk and culture: An essay on selection of technological and environmental danger. University of California Press.

Dubois, P. H. (1970). A history of psychological testing. Boston: Allyn and Bacon.

Dubowitz, H., Papas, M. A., Black, M. M., \& Starr, R. H. (2002). Child neglect: outcomes in high risk urban pre-schoolers. Pediatrics, 109(6), 1100-1107.

Escoriza, N. J. (1998). Conocimiento psicológico y conceptualización de las dificultades de aprendizaje. Barcelona: Ediciones de la Universitat de Barcelona. 
Espinosa, M., Colom, R., \& Quiroga, M.A. (1997). La práctica de la psicología diferencial en educación, clínica y deportes. Madrid: Pirámide.

Eysenck, H. J. (1989). El lugar de las diferencias Individuales en la Psicología Científica. Estudios de Psicología, 10(39-40), 161-206.

Forteza, J. (1993). Introducción. Investigaciones Psicológicas, 5, 7-16.

Giddens, A. (1990). Consecuencias de la Modernidad (1ra ed.). Barcelona: Alianza Editorial.

Glover, J. A., \& Bruning, R.H. (1990). Educational Psychology principles and applications (3ra ed.). Glenville, IL: Scott, Foresman.

Guilford, J. P. (1967). Creativity: Yesterday, today and tomorrow. The Journal of Creative Behavior, 1(1), 3-14. https://doi.org/10.1002/j.2162-6057.1967.tb00002.x

Hederich, C., \& Camargo, A. (2000) Estilo cognitivo y logro académico en la ciudad de Bogotá. Revista Colombiana de Educación, 40-41, 1-21. Recuperado de http://revistas.pedagogica.edu.co/index.php/ RCE/article/view/7782

Lluis, J. M. (2004). Mente y personalidad: Un nuevo modelo integrador. Madrid: Biblioteca Nueva.

López, F., Carpintero, E., \&Hernández, A. (2002). Prevalenciayconsecuencias del abuso sexual al menor en España. Child Abuse \& Neglect, 19(9), 1039-1050. https://doi.org/10.1016/0145-2134(95)00066-H

Ministerio de Salud de Chile (1998). Guía para la detección y respuesta al maltrato físico y abuso sexual infantil en los servicios de urgencias. Santiago: Unidad de Salud Mental.

Miras, M., \& Onrubia, J. (1996). Desenvolupament personal i educació. En C. Coll, Psicología de l’Educació. Barcelona. Universitat Oberta de Catalunya.

Moos, R. H. (1974). Psychological techniques in the assessment of adaptive behaviour. In Coelho G.V., Hamburg D.A. \& Adams J.E., (eds.), Coping and Adaptation. New York: Basic Books.

Moos R. H., (ed) (1976). Human Adaptation: Coping with Life Crises. Lexington: Heath.

Pellegrino, J.W. (1996). Abilities and Aptitudes. En E. de Corte y F.E. Weinert (Eds.), international Encyclopedia of Developmental and Instructional Psychology. New York. Pergamon Press. 
Radke, Y., \& Sherman, T. (1992). Hard growing: children who survive. En J. Rof, A. Masten, D. Cicchetti, K. Nuechterlein, \& S. Weintraub. Risk and protective factors in the development of psychopathology. New York, NY, US: Cambridge University Press.

Richters, J., \& Weintaub, S. (1992). Beyond disthesis: toward an understanding of high-risk environments. En J. Rof, A. Masten, D. Cicchetti, K. Nuechterlein, \& S. Weintraub. Risk and protective factors in the development of psychopathology. New York, NY, US: Cambridge University Press.

Robach, L. (1931), Behaviorism and psychology. Cambridge, Mass: Sci-art.

Ross, V., Marshall, M. H., \& Scott, A. M. (2001). Psicología Infantil (2da ed.). Barcelona: Ariel Psicología.

Rutter, M. (1987). Psychological Resilience and Protective Mechanisms. Am J Orthopsychiatry, 57(3), 316-331. https://doi.org/10.1111/j.1939-0025.1987.tb03541.x

Sánchez, A., \& Paniagua, E. (1996). Perspectivas actuales en el estudio de las diferencias individuales: Estructuras y procesos (2da ed.). Madrid: Sanz y Torres.

Santostefano, S., Quiroga-Estevez, M. A., \& Rooney-Santostefano, S. (2001). Life Stressors and cognitive styles in children. The Spanish Journal of Psychology, 4(1), 37- 50. https://doi.org/10.1017/ S1138741600005631

Snow, R.S. (1986). Individual differences and the desing of educational program. American Psychologist, 41(10), 1029-1039. https://psycnet.apa.org/doi/10.1037/0003-066X.41.10.1029

Smeby, J. (1996). Disciplinary differences in university Teaching. Studies in Higher Education, 21(1), 69-69. https://doi.org/10.1080/0307507 9612331381467

Sternberg, R.J. (1985). Implicit theories of intelligence, creativity and wisdom. Journal of Personality and Social Psychology, 49(3), 607-627. http://dx.doi.org/10.1037/0022-3514.49.3.607

Underwood, B. J. (1975). Individual differences as a crucible in theory construction. American Psychologist, 30(2), 128-134. http://dx. doi.org/10.1037/h0076759 